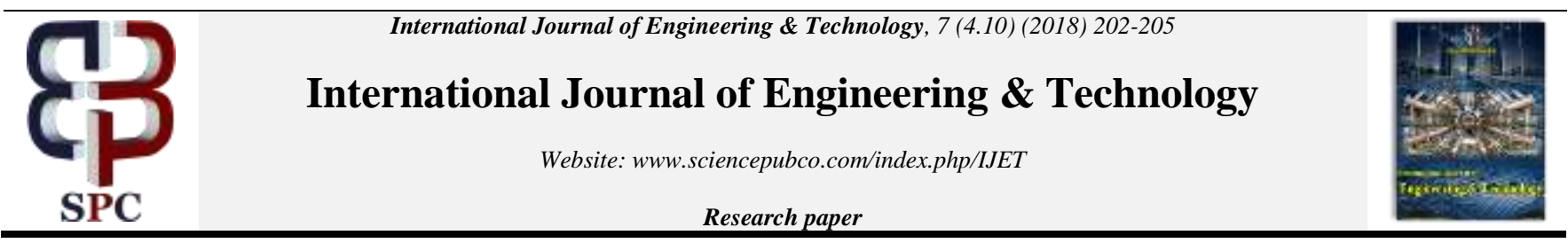

\title{
Performance Evaluation of Frame based Adaptive Compressed Sensing and Non-Adaptive Compressed Sensing based on Average Frame Signal to Noise Ratio
}

\author{
T. S. Arulananth ${ }^{1, *}$, R. Satheesh ${ }^{1}$, P. Bhaskara Reddy ${ }^{1}$ \\ ${ }^{1}$ Department of Electronics and Communication Engineering, MLR Institute of Technology, Hyderabad -43, Telangana, India \\ *Corresponding author E-mail: arulanandh.ts@gmail.com
}

\begin{abstract}
The primary inspiration of our work is to discovering upgrades in the current Compressed Sensing procedure that utilizations Non Adaptive Projection Matrix rule. Normal Frame Signal-to-Noise Ratio (AFSNR) is intended to evaluate the show of the Frame-Based Adaptive Compressed Sensing with the Non-Adaptive Compressed Sensing (CS). It is a developing sign securing strategy and straight gathers the signs in a compacted shape on the off chance that they are meager on some specific premise. Proposed approach utilizes Adaptive Projection Matrix in light of edge examination which gives fundamentally enhanced discourse recreation quality and decreases the noise levels.

Keywords: Adaptive sensing, Compressed sensing, non adaptive sensing, Nyquist rate, Signal to noise ratio
\end{abstract}

\section{Introduction}

Compressive Sensing (CS) is a rising and capable innovation that intensely understands the sparsity of the info signal. The primary target of Compressed Sensing must be utilized to redesign the signal accurately and effectively from little set non-versatile straight measured esteems. It is not a simple procedure to rebuild the subjective signs from a defective arrangement of direct measured esteems.

There is much essential conduct to be considered to recuperate the first signal. The calculation ought to be quick to recuperate signals viably. Most likely, slightest capacity necessities too would be an extreme point. The calculation ought to give uniform certification to recuperate every scanty signal without misfortune. Ideally, another calculation would require which requires just couple of straight estimations to recuperate the signs. Our prime necessities are to recoup the signal without noise.

\section{Existing System}

Existing frameworks are not giving the administration up to our normal level. Compressed Sensing (CS) is the system is utilized to conquer the formal imperatives of customary examining hypothesis. We all know that the sampling theorem says that the sampling frequency should be $\geq 2 \mathrm{f}_{\mathrm{m}}$.If we are not satisfied with this sampling condition it will leads to aliasing effect in the sampling process. But the compressed sensing allows us to go even beyond the Nyquist rate. The existing compressed sensing uses the non adaptive projection matrix and takes the same number of projections for all the frames. This may leads to degradation in system's efficiency.

\section{Proposed System}

Compressed Sensing (CS) is a rising trends and is focus in recent years for its simultaneous sampling and compression of sparse signals. Neighboring frames are compared to estimate their intraframe correlation, every frame is classified into different categories, and the number of projections for each frame is adjusted accordingly.

\section{Implementation of Adaptive Compressive Sensing Technique}

This segment we will talk about the execution of Adaptive Compressive Sensing (CS) strategy. Signal is created arbitrarily and thought to be settled amid the whole transformation process. Projection grid is dependably non-versatile in nature. This proposed procedure gives enhanced execution than the current calculation.

\subsection{The Adaptive Projection Matrix Concept}

By and large, Compressive Sensing strategy explores concentrates on irregular projection network calculation which is developed by considering just the signs sparsity instead of different properties. Something else, the development of projection grid is nonversatile. The neighboring casings are contrasted with estimated their intra-outline connection, each edge is grouped into various classes, and the quantity of projections is balanced in view of it. From the exploratory outcomes that the Adaptive Projection Matrix can impressively enhance the discourse change quality with less exertion. Intra-outline connection of discourse signals is investigated to accomplish effective testing. Distinctive kind discourse signs may have diverse intra-outline connections. AFBA Compressed Sensing system utilizes diverse testing approaches in various kind discourse outlines. 


\subsection{Frame Analysis of Adaptive Projection Matrix}

Every discourse grouping is isolated into covering outlines with the span of ' $1 \mathrm{x} \mathrm{n}$ ' and all edges in a discourse arrangement are handled freely. The projection framework is initialized by Gaussian irregular grid ' $\Phi$ ' which has been turned out to be ambiguous with most inadequate premise at high likelihood rate.

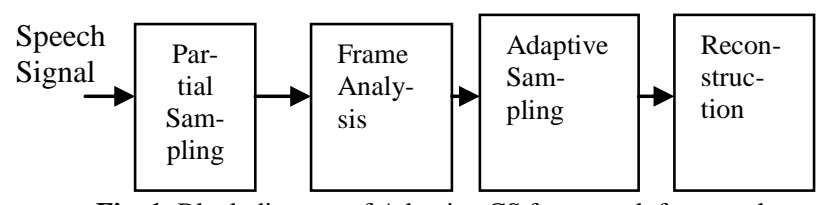

Fig. 1: Block diagram of Adaptive CS framework for speech

From Figure1, for each casing in a discourse arrangement, few projections is gathered, and contrasted these projections and the projections gathered for the past casing. In light of the examination comes about, the relationship between's these two edges is evaluated, and the connection is grouped into various classifications. At that point the testing technique is balanced by the connection sort and distinctive number of tests for the present edge is gathered.

The present casing of the genuine discourse signal is spoken to by $\mathrm{x}(\mathrm{t})$ and its past casing is spoken to by $\mathrm{x}(\mathrm{t}-1)$. The distinction between $x(t)$ and $x(t-1)$ mirrors the relationship between's the two neighboring edges, and can be utilized to group the connection. The gathered estimations are utilized to assess the relationship. A similar projection grid ' $\Phi$ ' is connected to all edges in the fractional testing stage, and after that $\mathrm{y}(\mathrm{t})-\mathrm{y}(\mathrm{t}-1)=\Phi \mathrm{x}(\mathrm{t})-\Phi \mathrm{x}(\mathrm{t}-1)$, where $y(t)$ and $y(t-1)$ are the projection vectors of $x(t)$ and $x(t-1)$ separately. As each specimen in $y(t)-y(t-1)$ is a direct blend of $x(t)$ $\mathrm{x}(\mathrm{t}-1)$ the distinction between the two projection vectors additionally mirrors the force changes in the two edge. The measure of power changes in the two edges can be assessed utilizing just few projections. Give ' $\Phi \mathrm{M} 0$ ' a chance to be a framework containing the primary ' $M$ ' 0 ' lines of the Gaussian irregular grid ' $\Phi$ '. For the current frame ' $t$ ', first ' $\Phi \mathrm{M}_{0}$ ' is used to collect ' $\mathrm{M}_{0}$ ' measurements $y(t) \wedge M_{0}=\Phi M_{0} x(t)$. Then, it is compared with the first ' $\mathrm{M}_{0}$ ' measurements in $\mathrm{y}(\mathrm{t}-1)$ and the difference $\mathrm{y}(\mathrm{t}) \wedge \mathrm{d}=\mathrm{y}(\mathrm{t}){ }^{\wedge} \mathrm{M}_{0}-\mathrm{y}(\mathrm{t}-$ 1) ${ }^{\wedge} M_{0}$ is calculated. In the frame analysis module, given $y(t) \wedge d$, its $1^{\wedge} 2$ norm normalized by $\mathrm{M}_{0}$ is calculated and compared with two thresholds $\mathrm{T} 1$ and $\mathrm{T} 2(\mathrm{~T} 1<\mathrm{T} 2)$.

On the off chance that $\mathrm{y}(\mathrm{t}) \wedge \mathrm{d} / \mathrm{M}_{0}<=\mathrm{T} 1$, the present casing is nearly the same as its past edge. The two neighboring casings are considered might be both surd and the intra-outline connection is named as surd versus surd. On the off chance that $\mathrm{T} 1<\mathrm{y}(\mathrm{t}) \wedge \mathrm{d} / \mathrm{M}_{0}$ $<=\mathrm{T} 2$, it shows that these two neighboring edges experience little changes. In this circumstance, the two neighboring edges might be both sonant at high likelihood and the intra-connection is named as sonant versus sonant. On the off chance that $y(t) \wedge d / M_{0}>T 2$, the two edges are essentially not quite the same as each other, which is no doubt because of the difference in the edge sort, and the connection is diversely marked as surd versus sonant.

\subsection{Partial Sampling Approach}

Each casing in a discourse grouping, few projections is gathered and that is contrasted and the modest number of projections gathered for the past edge. In view of the examination comes about, the relationship between's these two edges is assessed, and the connection is characterized into various classes. At that point the testing system is balanced by the connection sort and distinctive quantities of tests are gathered for the present edge.

\subsection{Adaptive Sampling Approach}

Contingent upon their ordered intra-outline connection sorts, diverse number of projections is utilized for the discourse outlines. The casing is considered as surd casing if its intra-outline connec- tion sort is surd versus surd. A surd casing contains the slightest new data in the discourse. Therefore, the $\mathrm{M}_{0}$ estimations gathered in the halfway examining stage are adequate and extra inspecting does not required. At the point when its intra-outline relationship is sonant versus sonant, the casing is considered as sonant and contains some new data, which requires more estimation to be gathered.

For such edges, $(\mathrm{M} 1>\mathrm{M} 0)$ estimations are gathered. The $\left(\mathrm{M}_{0}+1\right)^{\mathrm{th}}$ to the $M 1^{\text {th }}$ columns of the Gaussian irregular lattice ' $\Phi$ ' are utilized and joined with M0 to create the last projection vector. The casings that experience substantial changes must contain the most new data. Consequently, an aggregate of M2 (M2>M2>M2) estimations are gathered amid the testing procedure. The aggregate projection grid is the primary M2 lines of the Gaussian arbitrary framework ' $\Phi$ '.

$$
\sum_{\mathrm{K}=1}^{\mathrm{k}} 10 \log _{10}\left(\left\|\mathrm{x}_{\mathrm{k}}\right\|\right)^{2} /\left(\left\|\mathrm{x}_{\mathrm{k}}-\mathrm{x}_{\mathrm{k}},\right\|^{2}\right)
$$

\section{Results and Discussion}

When we looking at the show of our proposed calculation with normal Compressive Sensing. We can watch that radical changes in reproduced come about. For that perception, a discretionary discourse signal has been picked with the reasonable Threshold esteems 'T1' and 'T2' are as 0.08 and 0.4 individually and that is tested through a lot of cycles. The Average Frame Signal to Noise Ratio (AFSNR) is watched, classified and used to assess the recreation nature of discourse signal. Normal Frame Signal to Noise Ratio (AFSNR) is computed utilizing the recipe AFSNR $=1 / \mathrm{K}$. Where ' $K$ ' is the aggregate number of casing of a discourse signal and $\mathrm{xk}$ and $\mathrm{xk}^{\prime}$ are speaks to the $\mathrm{k}^{\text {th }}$ edge of the discourse and the $\mathrm{k}^{\text {th }}$ outline remade discourse individually with various packed proportion, we organized the outcomes. Where ' $r$ ' is the compression proportion it is characterized as $r=M / N$. Where ' $M$ ' is the quantity of projections and ' $\mathrm{N}$ ' the edge per tests.

\section{Input Signal}

The information signal which was at that point recorded is a blend of voiced and unvoiced sounds, and it goes on for seven seconds. Examining recurrence of the information signal is $8 \mathrm{kHz}$, and each specimen is 8 bit encoded. So the bit rate of the signal is $64 \mathrm{kbps}$. Add up to no of tests show in the signal is equivalent to the result of examining and aggregate span of the signal in seconds. Consequently, add up to no of tests $=8000 * 7=56,000$. The information signal is experienced through Discrete Cosine change (DCT) method which makes the signal meager. The signal which is gotten by applying DCT to unique info signal is as yet containing some low coefficients with low sufficiency esteems. These coefficients must be expelled to influence the signal to advance scanty. Subsequently the signal is experienced through a sifting procedure that disposes of the coefficients whose sufficiency is beneath 0.04 or more -0.06 .

\section{Frame Analysis}

The separated signal is sufficiently meager to apply the packed detecting. The entire signal is separated into 175 non covering outlines keeping in mind the end goal to apply the Adaptive Compressed Sensing. Each edge comprises of 320 specimens.

\section{Projection Matrix}

Projection network is a haphazardly created lattice in which the no of segments is equivalent to ' $\mathrm{N}$ ', and the no of columns is equivalent to ' $\mathrm{M}$ '. Where $\mathrm{N}$ is equivalent to the no of tests display in the single edge, for this situation the edge estimate is 320 and consequently $\mathrm{N}=320 . \mathrm{M}$ is equivalent to $\mathrm{r} * \mathrm{~N}$, where ' $r$ ' is the compression ratio.

For compression ratio $\mathbf{r}=\mathbf{0 . 2} \mathrm{N}=320, \mathrm{r}=\mathrm{M} / \mathrm{N}=0.2$. Hence $\mathrm{M}=\mathrm{r} * \mathrm{~N}=0.2 * 320=64$

For compression ratio $\mathbf{r}=\mathbf{0 . 4} \mathrm{N}=320, \mathrm{r}=\mathrm{M} / \mathrm{N}=0.4$. Hence $\mathrm{M}=\mathrm{r} * \mathrm{~N}=0.4 * 320=128$ 
For compression ratio $\mathbf{r}=\mathbf{0 . 6} \mathbf{N}=320, \mathrm{r}=\mathrm{M} / \mathrm{N}=0.6$. Hence $\mathrm{M}=\mathrm{r} * \mathrm{~N}=0.6 * 320=192$

Compressed Sensed Signal

The Compressed Sensed signal is acquired by duplicating the info outline with the projection grid relating with the required compression proportion. Compression Ratio ' $r$ ' $=0.2$ when the quantity of projections ' $\mathrm{M}$ ' $=64$ for the edge of tests ' $\mathrm{N}$ ' $=320$. The PC produced result is appeared in underneath figure 2, 3 and 4 . An outcome demonstrates the time area qualities of the interesting discourse signal. AFSNR for compression proportion $r=0.2$ with versatile projection lattice is 12.030 which is 4.970 with non versatile projection grid. Here we can infer that the AFSNR is expanded by Adaptive Projection Matrix sensibly.

\section{Case (i) AFSNR for ' $r$ ' $=0.2$}
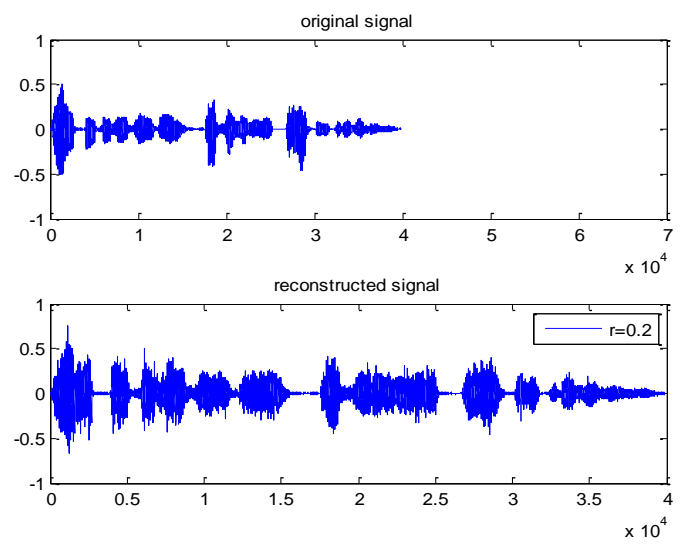

Fig. 2: Original speech signal \& Reconstructed signal with compression ratio

$\mathrm{r}=0.2$

\section{Case (ii) AFSNR for ' $r$ ' $=\mathbf{0 . 4}$}

At the point when the compression proportion ' $r$ ' $=0.4$ which demonstrates that the quantity of projections 'M'=128 for the edge of tests ' $\mathrm{N}$ ' $=320$. The figure 3 show the time area attributes of the genuine discourse signal and Adaptive CS reproduced discourse with ' $r$ ' $=0.4$.The AFSNR for compression proportion $r=0.4$ with versatile projection grid is 15.1714. Furthermore, which are 12.103 just with non versatile projection framework. Hence the AFSNR can be expanded by versatile projection framework. From the above figure 3 , it is watched that, when the packed proportion $r=0.4$, the nature of the remade signal has been expanded than the recreated signal got from compacted proportion $\mathrm{r}=0.2$. The last perception is AFSNR differing specifically relative to the compression proportion ' $r$ '.
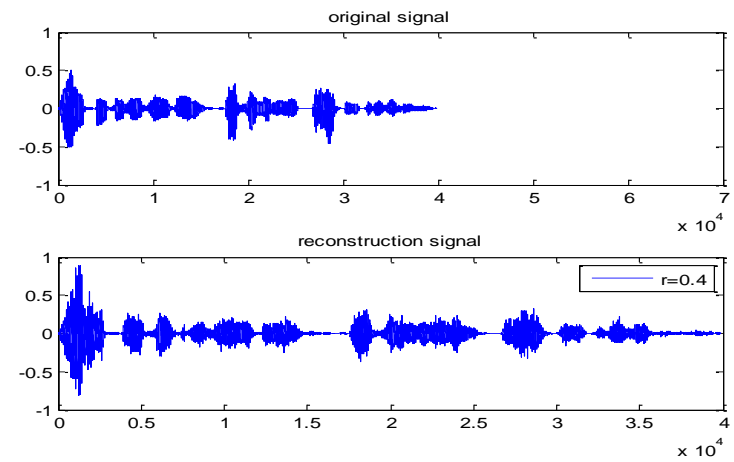

Fig. 3: Original speech signal \& Reconstructed signal with compression ratio $=0.4$

Case (iii) AFSNR for compression ratio $r=0.6$

For this situation the compression proportion ' $r$ ' $=0.6$ shows the quantity of projections ' $\mathrm{M}$ ' $=192$ for the ' $\mathrm{N}$ ' $=320$. The figure4 demonstrates the time area waveform of the first discourse signal and Adaptive CS reproduced discourse with compacted proportion of 0.6.The AFSNR for ' $r$ ' $=0.6$ with versatile projection framework is 25.1721 which is 21.630 with non Adaptive Projection lattice. Along these lines the AFSNR can be expanded by versatile projection grid. From the figure4, it is watched that, when the packed proportion $\mathrm{r}=0.6$, the nature of the reproduced signal has been expanded than the remade signal acquired from compacted proportion of lesser esteems like $r=0.2$ and $r=0.4$.
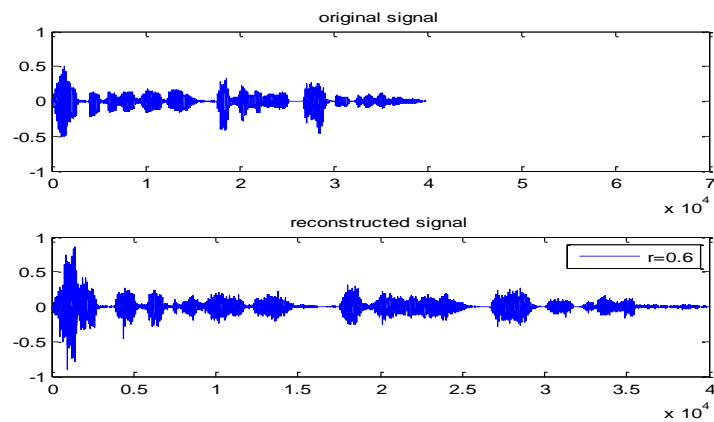

Fig. 4: Original signal \& Reconstructed signal with Compression ratio $\mathrm{r}=$ 0.6

Comparison of AFSNR for adaptive CS and non adaptive CS The outcomes got by utilizing Adaptive Projection Matrix to actualize the Compressed Sensing are contrasted and that of unsurprising non versatile packed detecting, for the three compacted ratio ' $r$ ' $=0.2,0.4$ and 0.6 .

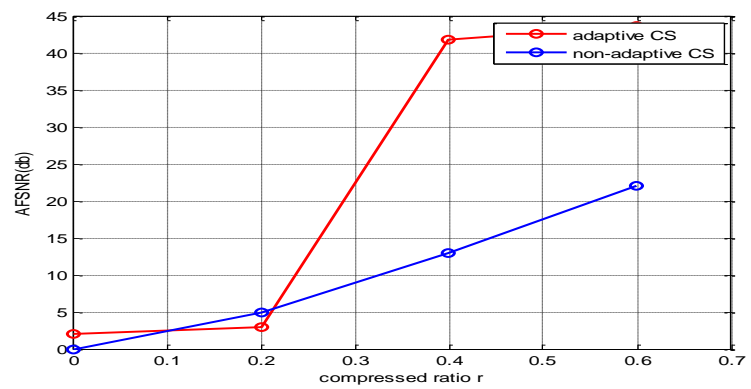

Fig. 5: AFSNR Comparison of Adaptive CS and non adaptive CS

From figure 5. We can watch that the nature of reproduced discourse signal in view of Frame-based Adaptive CS and the nonversatile CS. At the point when the packed proportion is ' $r$ ' $=0.4$, the AFSNR expands more than $7 \mathrm{~dB}$. Table 1 gives the Average Frame Signal to Noise Ratio (AFSNR) of the remade voice in view of our proposed calculation and the ordinary CS. For the diverse estimations of ' $r$ ', the reproduced discourses are arranged.

Table 1: AFSNR of Conventional and Adaptive CS

\begin{tabular}{|l|l|l|l|}
\hline Compression ratio & 0.2 & 0.4 & 0.6 \\
\hline Non Adaptive CS & 5.00 & 13.00 & 22.00 \\
\hline Adaptive CS & 2.9185 & 41.7593 & 43.6308 \\
\hline
\end{tabular}

Thus it is clearly indicates that the proposed Adaptive Compressed Sensing is more efficient than the conventional non adaptive compressed sensing.

\section{Conclusion}

The Average Frame Signal to Noise Ratio has been enhanced by utilizing Adaptive Projection Matrix. That is demonstrated by the test comes about, that the nature of the rebuilt signal is increments when the packed proportion ' $r$ ' increments. In this manner the current framework can be supplanted by the Adaptive Compressed Sensing to propel the proficiency of the framework. Distinctive tests and investigation is utilized to locate the sufficiently most enhancement strategy to remake the discourse signal. Despite the 
fact that this method utilizes less estimations does not losing any of the data.

\section{References}

[1] Donoho D L. J. Tropp, A Gilbert (2006) Signal recovery from random measurements via orthogonal compressed sensing. IEEE Transaction on information theory, 6, 52(4), 1289-1306.

[2] Donoho D, Tsaig Y (2006) Extensions of compressed sensing, signal processing, 86(3), 533-548.

[3] Scott S.chen, David L.Donoho, Michel A .Saunders (2001).Atomic decomposition by basis pursuit. Society for industrial and applied mathematics, SIAM review, 43(1):129-159.

[4] Zh. M Wang, G. R. Arcet, J. L. Paredest (2007). Colored random projections for compressed sensing, ICASSP, 873-876.

[5] Zhaorui Liu, Vicky Zhao, A. Y. Elezzabi (2010). Block-based adaptive compressed sensing for video, Proceedings of 2010 IEEE 17th international conference on image proceeding, 1649-1652.

[6] Jarvis haupt, Rebert Nowak, Rui Castro (2009). Adaptive sensing for sparse signal recovery. ICASSP, 1, 702-707. 